\title{
Moderate contact between sub-populations promotes evolved assortativity enabling group selection
}

\author{
James R. Snowdon, Simon T. Powers, and Richard A. Watson \\ School of Electronics and Computer Science, University of Southampton, U.K.; \\ raw@ecs.soton.ac.uk
}

\begin{abstract}
Group selection is easily observed when spatial group structure is imposed on a population. In fact, spatial structure is just a means of providing assortative interactions such that the benefits of cooperating are delivered to other cooperators more than to selfish individuals. In principle, assortative interactions could be supported by individually adapted traits without physical grouping. But this possibility seems to be ruled-out because any 'marker' that cooperators used for this purpose could be adopted by selfish individuals also. However, here we show that stable assortative marking can evolve when sub-populations at different evolutionarily stable strategies (ESSs) are brought into contact. Interestingly, if they are brought into contact too quickly, individual selection causes loss of behavioural diversity before assortative markers have a chance to evolve. But if they are brought into contact slowly, moderate initial mixing between sub-populations produces a pressure to evolve traits that facilitate assortative interactions. Once assortative interactions have become established, group competition between the two ESSs is facilitated without any spatial group structure. This process thus illustrates conditions where individual selection canalises groups that are initially spatially defined into stable groups that compete without the need for continued spatial separation.
\end{abstract}

\section{Introduction}

The perspective of group selection is often used to explain altruistic behaviour. Sober and Wilson provide the definition that 'a behaviour is altruistic when it increases the fitness of others and decreases the fitness of the actor' [1], which can appear unsupportable by traditional theories of natural selection as echoed by Dawkins [2], who claims that for any gene to survive they must promote themselves at the expense of others. A gene which supports altruistic behaviour would quickly be exploited to extinction by selfish cheaters.

As such selfishness can be described as an Evolutionarily Stable Strategy (ESS). A behaviour is considered to be an ESS if when all individuals within a population adopt a particular behavioural strategy no other type can successfully invade [3]. Altruism is therefore not an ESS since it can be invaded by cheats who will exploit the benefit awarded to them and not reciprocate. For natural 
selection to occur at any level, there must exist a fitness variance between entities [4], where an entity can be an individual or a group composed of individuals forming a meta-population. In this one ESS system all groups will move towards a situation where every individual holds the same behaviour, resulting in no variance for natural selection to act upon.

Wilson [5] uses groups comprised of altruists and cheaters to provide a setting in which altruism can prevail. Each group reproducing individually would ultimately be drawn towards the one stable attractor which is the all selfish ESS, but groups are dispersed prior to this occurring and the progeny are mixed. New groups are composed of random samples and the aggregation and dispersal process is repeated indefinitely. Since groups which contain more altruists grow at a faster rate than groups composed of majority cheats, the effect is that the net proportion of altruists rises. However, when practically assessing the model it is found that between group variance in the frequencies of cheats and selfish types is required to be extremely high, and as such a stronger effect is observed through taking an extremely small sample of the population when creating each new group in the aggregation process. This limit of small group sizes coupled with the aggregation and dispersal movement limits the applicability to real world situations. Although the conditions for such altruism to evolve are restrictive, Powers et al. [6] have investigated how these conditions can in fact arise by evolution of individual traits that modify aspects of population structure, such as group size.

Wilson [7] studies group level selection in complex meta-communities. His model shows how individuals detrimental to local community productivity can be purged through selection at the community level. This is an example of a multi-ESS system, for the internal community dynamics have many attractors (ESSs) and proves to be more effective than previous altruist/ cheat models, since between-group variance could be preserved even when the groups reached an internal equilibrium. Such a form of group selection is also explored by Boyd and Richerson who consider selection acting among multiple ESSs [8], where each group reaches an ESS holding an inherent fitness thus providing a between group variance. Through competition with other groups based upon a migratory process, the ESSs compete and some are forced into extinction as groups reach the point of highest individual fitness. Their model does not consider the forces which give rise to these groupings, even though they are able to illustrate the selection processes which act at the group level.

We are interested in situations where a form of group selection can occur which is not restricted by the need for constant spatial segregation. Consider a scenario where two spatially separated sub-populations develop different behaviours, A and B, which are both ESSs. The sub-populations are then slowly brought into contact, perhaps through natural expansion of each of the subpopulations. One of these behaviours, say A, even though it may have been intrinsically fitter than B, may be lost when the two populations are brought into contact because it fares poorly in interaction with B, because B may be more numerous. In principle, if individuals within the two sub-populations evolved 
distinguishable markers, such as the secretion of a unique, identifiable chemical which correlated with behaviour and promoted assortative interactions then competition between the groups would be enabled when the two are brought into contact. That is, although A loses to B, A-A wins in interaction with B-B. This would mean that the two sub-populations had formed higher-level units of selection.

A model provided by McElreath, Boyd and Richerson [9] in the domain of cultural evolution shows that individual selection can evolve markers that are correlated with behaviour and promote assortative interactions. Their work does not address the notion that such marking facilitates higher-level selection - they are interested in the promotion of stably coexisting (ethnically marked) groups. But we show that the conditions they illustrate for evolving behaviourally-correlated markers are also suitable to thereby facilitate inter-group competition.

\section{A model of the evolution of assortative markers under some degree of spatial segregation}

Our model is founded upon the work of McElreath et al [9]. We describe individuals as exhibiting a behaviour, labelled A and B, and a 'marker' trait, labelled 1 and 2 . This can be interpreted as an externally apparent phenotype used for the purpose of facilitating assortative interactions (as per Boyd et al's work [10]) or any other trait which has the effect of producing assortative interactions, such as a habitat preference [11].

Upon initialization individuals are distributed between two sub-populations, and we start from the scenario where each is already at a stable A or B ESS. The algorithmic operation of the model for each subsequent time step is as follows:

1. Interactions - All individuals interact with each other within the same subpopulation in a coordination game with a payoff matrix as shown in table 1.

Table 1. Payoff matrix for interactions between individuals harbouring either behaviour A or behaviour B. This system exhibits both A and B as ESSs.

\begin{tabular}{l||l||l||} 
& $\mathrm{A}$ & $\mathrm{B}$ \\
\hline $\mathrm{A}$ & $1+\delta+\alpha$ & 1 \\
\hline $\mathrm{B}$ & 1 & $1+\delta$ \\
\hline
\end{tabular}

An individual interacting with another holding the same behavioural trait will enjoy an advantage, $\delta$ but only the marker can influence which other individual is likely to be interacted with. A parameter $e$ describes assortativity, or marker strength - the probability that an individual will interact with another possessing the same marker. When $e=1$ individuals will only interact with others of the same marker as themselves, and when $e=0$ individuals interact totally randomly with no reliance upon marker. 
2. Reproduction - The proportions, $N$, of each marker, $i$, and behaviour, $j$, within the next generation of individuals changes according to equation 1 , where $\sum W_{i j}$ is the total payoff awarded to each behaviour/marker type (A1, $\mathrm{A} 2, \mathrm{~B} 1$ and $\mathrm{B} 2)$ and $\bar{W}_{i j}$ is the average payoff awarded to individuals of that type.

$$
N_{i j}=\frac{\sum W_{i j}}{\bar{W}_{i j}}
$$

3. Migration - Prior to being brought together, there is a degree of migration between the two groups, represented by the parameter $m$. This relocates a random proportion of each group to within the other, thus representing a metric of spatial segregation. Groups at a value of $m=0$ implies that no migration exists as groups are completely segregated, and a larger value of $m$ (maximum 0.5) represents a freely mixed population.

In order to generate a between group fitness variance, A-A interactions are given an additional payoff bonus $\alpha$, and groups are brought into a competitive state by bringing the sub-populations together after a fixed period by setting the inter-group migration to a maximum, $m=0.5$.

\section{Exploring the behaviour of two sub-populations}

It would of course be possible to set up the initial conditions of the two groups such that markers correlated with behaviours will be reached regardless of interaction. However, we set the initial conditions of the sub-populations such that they have different behaviours in the majority and the same marker in the majority. This means that without some selective pressure to cause markers to diversify, both groups will have the same marker and assortative interactions will not be possible. We also set the size of one sub-population to be slightly larger than the other - the sub-population with the inferior behaviour - for similar reasons. That is, we want to identify conditions where the superior behaviour prevails despite being initially disadvantaged. We will refer to this correlation of marker/ behaviour pairs as the evolution of assortative markers.

Table 2. Parameters used for the simulation process

\begin{tabular}{|l|c|l|l|c|}
\hline Parameter & Value & Parameter & Value \\
\hline Sub-population 1 Prop. 1 Marker & 0.9 & Sub-population 2 Prop. 1 Marker & 0.6 \\
Sub-population 1 Prop. 2 Marker & 0.1 & Sub-population 2 Prop. 2 Marker & 0.4 \\
Sub-population 1 Size & 2000 & Sub-population 2 Size & 3800 \\
Like-for-like payoff, $\delta$ & 0.5 & A-A payoff bonus, $\alpha$ & 0.1 \\
\hline
\end{tabular}


The simulation was run with the initial parameters shown in table 2 , and as will be seen the values of $e$ and $m$ will come under further investigation so these are not fixed.

Crucially groups are initialised such that if the initial migration rate is too high then the B behaviour will overwhelm as before markers have evolved in the system due to the larger sub-population 2 holding a higher majority of B type. If the migration rate is too low then assortative markers will not evolve despite sub-populations being behaviourally marked. Under these conditions should assortative marking occur, if one of the sub-populations was going to go to the marker 1, we would expect it to be sub-population 1 .

When groups are freely mixed after an initial partial mixing phase, we would expect the ultimate results to be predictable through observing the basins of attraction shown in figure 1. If markers have not been able to evolve (for example from a zero migration rate) then we would expect the distribution of each marker type in a sub-population to be random as frequencies would drift. The ultimately winning strategy would be B as when the sub-populations are mixed, the majority - $65 \%$ - of the newly formed group would be from the all-B group 2 , and this would overwhelm any advantage which A-A receives which would be unable to successfully interact assortatively.

If the migration rate is too high then all individuals in both sub-populations will become type $\mathrm{B}$ due to the initial majority of behaviour Bs in the entire population, and so the mixed group would then already be at the all-B attractor. However, if assortative markers have evolved then at $e=0.5$ despite only $35 \%$ of the mixed group being A1 type, the resultant attractor is expected to be A1 as figure $1(\mathrm{~b})$ shows.

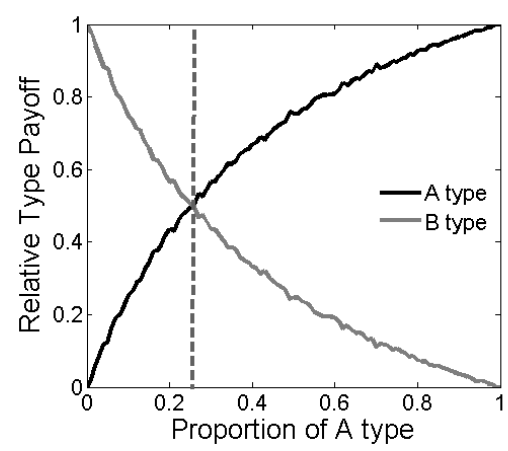

(a) Full correlation between behaviour and marker $(e=1)$

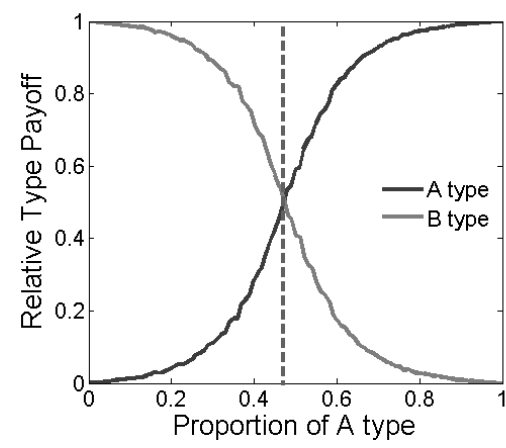

(b) No correlation between behaviour and marker $(e=0)$

Fig. 1. Relative fitness of A1 and B2 type when in competition in a single population. The line indicates the lowest initial proportion of A1 type required for A1 to reach fixation 
Figure 2 illustrates system behaviour for a fixed $e=0.5$ and varying migration rates. Figure 2(a) introduces a metric of the polarisation of marking present within the sub-populations as the linkage disequilibrium of marker and behaviour after an appropriate period of mixing. A higher value shows assortative markers have evolved, and 0 shows that such marking has not occurred. It is clear that a region exists where a degree of spatial segregation enables the evolution of such assortative marking.

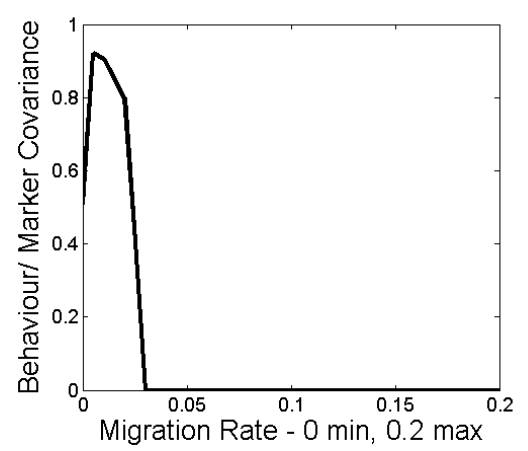

(a)

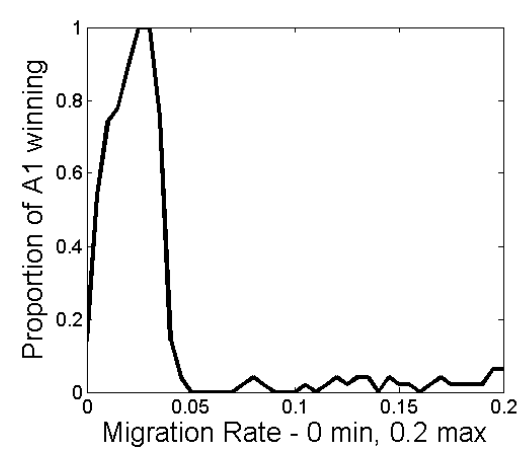

(b)

Fig. 2. a) Analysis over a range of spatial segregation values, when $e=0.5$, displaying marker/ behaviour covariance evolved with spatial segregation after 600 time steps, averaged over 20 runs b) Resulting proportion of A1 types which went on to reach fixation determined from an average of the same 20 runs

Figure 2(b) shows the proportion of runs when the groups were then brought together and the A1 behaviour type went on to reach fixation and drive the B2 behaviour extinct. Again we see that there is a region where A1 is able to outcompete B2 - and this corresponds to the region of polarised markers.

Taking single points from the graph in figure 3, we can observe the internal behaviour between the two sub-populations which leads to the results shown:

- No initial contact, $m=0.0$, shows that because the migration rate is zero there is no pressure for markers to evolve, so when the groups are brought together the effect is that B behaviour types overwhelm A behaviour.

- Moderate contact, $m=0.01$, shows that although sub-population 2 starts with marker 1 in the majority, there is a pressure not to interact with migrants of majority A1 type from sub-population 1. This causes marker 2 to cross over and become marked with behaviour B. When sub-populations are freely mixed this results in A1 winning.

- High contact, $m=0.2$, shows that B behaviour fixates within the entire population due to the higher migration rate, and again there is no pressure for markers to evolve. Accordingly the resultant 'winner' is non-polarised behaviour B. 


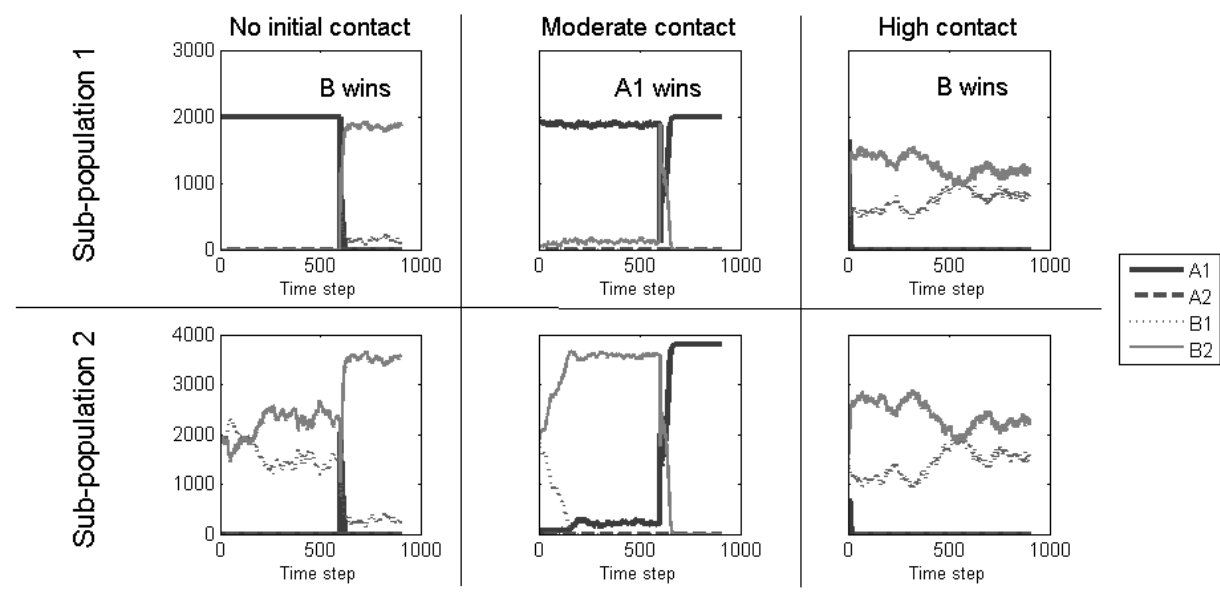

Fig. 3. Illustrating interactions with varying initial contact between sub-populations. Sub-population 1 (above) is fixed at size 2000, and sub-population 2 (below) is fixed at size 3800 . At $\mathrm{t}=600$ the sub-populations are mixed through setting inter-group migration to maximum, resulting in the domination of one behaviour

\section{Discussion}

We have shown that, as per McElreath et al's work, a small amount of initial mixing between sub-populations that exhibit different behaviours can produce a selective pressure to favour the evolution of markers that are correlated to those behaviours. No mixing and the markers have no function, too much mixing and one of the behaviours is lost before markers evolve; but a period with a small amount of mixing produces this effect. In essence, this occurs because it enables a period where weak indirect selective pressures on markers can be felt, whilst precluding the strong selective pressures on behaviours that would lose diversity before the markers have evolved. This effect means that partial spatial segregation between sub-populations (but not complete segregation) enables individuals to evolve behaviours that reinforce within-group interactions and we show that the assortative interactions that result facilitate competition between groups when increased mixing occurs. The evolution of markers in this way can be seen as construction of an individual's social environment [12]. Group selection can be facilitated by such a process, as shown here and in other work $[13$, 14].

In conventional altruist/ cheat dynamics involving the evolution of assortative markers there exists a possibility of cheats evolving the phenotypic altruistic marker, such as a green beard [2], signalling altruistic intent without actually posessing a cooperative gene. Because this model holds multiple stable ESSs 
the issue of cheating does not occur, as an individual falsely advertising its' behaviour would receive a lower payoff than if it had been honest.

This model illustrates very simple conditions where individual selection can favour the evolution of traits that support between-group competition. Accordingly, from one point of view, the expectation that individual selection cannot create significant group selection is shown to be false. But, from another point of view, one which takes into account both individual selection on markers and behaviours under these spatial conditions - individual selection explains the outcomes we observe. Indeed, if there were not the case, we would not have provided an evolutionarily explanation at all. Nonetheless, because the markers are different from behaviours in that they only have fitness consequences via their indirect effects on the assortativity of behaviours - we argue that a two scale selection theory is conceptually useful.

\section{References}

1. Sober, E., Wilson, D.S.: Unto others: the evolution of psychology and unselfish behavior. Harvard University Press (1998)

2. Dawkins, R.: The Selfish Gene. Oxford University Press (1976)

3. Maynard Smith, J,: Evolution and the Theory of Games. Cambridge: Cambridge University Press (1982)

4. Wilson, D.S.: Sober, E. . Reintroducing group selection to the human behavioral sciences. Behavioral and Brain Sciences 17 (4): 585-654. (1994)

5. Wilson, D.S.: A theory of group selection. PNAS 72(1) 143146 (1975)

6. Powers, S. T. and Watson, R. A. (2009). Evolution of individual group size preferences can increase group-level selection and cooperation. In Proceedings of the 10th European Conference on Artificial Life. To appear.

7. Wilson, D.S.: Complex interactions in metacommunities, with implications for biodiversity and higher levels of selection. Ecology, 73: 1984-2000 (1992)

8. Boyd, R., Richerson, P.J.: Group Selection among Alternative evolutionarily Stable Strategies. J. theor. Biol. 145, 331-342 (1990)

9. McElreath, R., Boyd, R., Richerson, P.J.: Shared norms and the evolution of ethnic markers. Current Anthropology 44(1) 122-129 (2003)

10. Boyd, R., Richerson, P.J.: Culture and the Evolutionary Process. University of Chicago Press (1985)

11. Wilson, D.S., Dugatkin, L.A.: Group selection and assortative interactions. Am Nat 149336 - 351 (1997)

12. Powers, S.T., Mills, R., Penn, A.S., and Watson, R.A. (2009). Social Environment Construction Provides an Adaptive Explanation for New Levels of Individuality. In Proceedings of ECAL 2009 Workshop on Levels of Selection and Individuality in Evolution: Conceptual Issues and the Role of Artificial Life Models.

13. Mills, R. and Watson, R. A. (2009). Symbiosis enables the evolution of rare complexes in structured environments. In Proceedings of the 10th European Conference on Artificial Life. To appear.

14. Watson, R. A., Palmius, N., Mills, R., Powers, S. T., and Penn, A. S. (2009). Can selfish symbioses effect higher-level selection? In Proceedings of the 10th European Conference on Artificial Life. To appear. 